\title{
Nanotecnología: Un nuevo paradigma científico en la producción agropecuaria del siglo $\mathbf{X X I}$
}

\section{Nanotechnology: A new scientific paradigm on agricultural production of the $X X I$ century}

\author{
Ricardo Hugo Lira-Saldivar ${ }^{1 *}$, Bulmaro Méndez-Argüello ${ }^{1}$ \\ ${ }^{1}$ Departamento de Plásticos en la Agricultura. Centro de Investigación en Química Aplicada (CIQA), Saltillo, Coahuila, \\ México. CP. 25294 \\ *Autor de correspondencia: hugo.lira @ciqa.edu.mx
}

Carta al editor recibida: 10 de junio de 2017 aceptada: 31 de octubre de 2017

El avance de la ciencia está llevando a la humanidad a la era de la nanotecnología (NT), que en términos sencillos, se refiere a la tecnología practicada sobre los materiales a nivel molecular. La NT, es la ciencia que está sustentada en la capacidad de medir, manipular y organizar la materia a escala nanométrica, entre 1 y 100 nanómetros $\left(10^{-9} \mathrm{~m}\right)$, tiene la capacidad de impulsar el incremento de la producción de alimentos por medio de la agricultura sustentable, debido a que permite usar agua, pesticidas y fertilizantes de forma más eficiente y en menor cantidad (Duhan et al. 2017). Las aplicaciones de la NT son enormes, como los nanosensores para el monitoreo fitosanitario, zeolitas nanoporosas para la liberación lenta de fertilizantes, nanoaditivos alimenticios, medicamentos, nanoemulsiones para el tratamiento y la prevención de enfermedades (Ganesh 2017). En el sector agrícola los reportes sobre la aplicación de nanopartículas (NPs) metálicas con $\mathrm{Fe}, \mathrm{Cu}$ y $\mathrm{Zn}$, indican que mejoran la germinación de las semillas, el crecimiento, el contenido nutricional, la actividad enzimática y el rendimiento (Bradfield et al. 2017).

Algunas NPs de $\mathrm{ZnO}$ y $\mathrm{CuO}$ muestran efecto positivo sobre la reactividad de fitohormonas como las citoquininas (zeatina), auxinas (ácido indolacético) y ácido salicílico, compuestos que promueven elongación, división celular y crecimiento en las plantas (Lira-Saldivar et al. 2016). Algunas respuestas a las NPs podrían estar relacionadas con la producción del esteroide diosgenina y precursores hormonales, que contribuyen en la producción endógena de fitohormonas como las citoquininas, auxinas y giberelinas (Vankova et al. 2017). Las NPs derivadas del carbono (C) como grafeno, fullereno y nanotubos de carbono (NTC) también son de importancia en la agricultura, debido a que tienen un efecto estimulante del crecimiento. Los NTC promueven el crecimiento de las plantas debido a que los nanotubos se comportan como canales de agua similares a las acuaporinas, lo que promueve mayor presión hidrostática dentro de las células; induciendo absorción de agua, flujo de nutrientes y por consiguiente mayor crecimiento de la planta.

Las NPs de óxido de grafeno también mejoran el crecimiento y la producción de biomasa en plantas, mientras que otras NPs como las de óxido de titanio causan incrementos en la actividad de varias enzimas y promueven la absorción de nitrato, el cual acelera la transformación del nitrógeno inorgánico a orgánico, haciéndolo más asimilable para las plantas. Un estudio con NPs de óxido de cerio demostró que también incrementan la producción de biomasa fresca, longitud de raíz y la actividad enzimática del sistema antioxidante (Gui et al. 2017). Lo que evidencia que las plantas cultivadas responden de forma favorable a los NPs metálicas o derivadas del carbón, y aun cuando algunos de estos nanocompuestos no actúan como micro o macro nutrientes, impactan en la fisiología y bioquímica de las plantas, efectos que no están bien dilucidados.

En la producción animal, los nanoaditivos en 


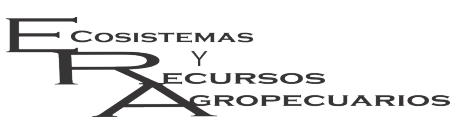

las dietas promueven el crecimiento, la salud y la calidad de la carne. Los nanomateriales en nutrición animal y medicina veterinaria apenas se están investigando, sin embargo, su aplicación ha aumentado, y se prevé que siga creciendo en el futuro (Swain et al. 2016). Las NPs han empezado a utilizarse para el suministro eficiente de nutrientes y como antimicrobianos de nueva generación. Minerales como el $\mathrm{Se}, \mathrm{Cu}, \mathrm{Fe}$ y $\mathrm{Zn}$ de tamaño nanométrico incorporados a las dietas de animales, pueden incrementar su absorción y bioutilización, lo que puede promover el crecimiento de los animales y mejorar la calidad de la carne (Hill y Li 2017). La NT en el futuro cercano promete revolucionar la industria agroalimentaria, de manera que aquellos países que no logren incorporarse a esta megatendencia, pueden quedar fuera de la nueva redistribución industrial resultante
Lira-Saldivar y Méndez-Argüello et al. Nanotecnología en producción agropecuaria

Ecosist. Recur. Agropec. 5(13):1-2,2018

de la competencia alimentaria.

En este contexto la NT es una necesidad urgente para cambiar los métodos y técnicas de producción de alimentos, y México la ha aceptado para su desarrollo; prueba de lo antes señalado es que el Centro de Investigación en Química Aplicada en coordinación con la Universidad Autónoma Agraria Antonio Narro, realizaron el International Symposium on AgroBio Nanotechnology del 25 al 27 de octubre de 2017 en Saltillo, Coahuila, México. Es importante resaltar que la NT es el conocimiento emergente del siglo XXI en todos los campos de la ciencia, y estamos convencidos que sus diversas aplicaciones en la agricultura y la ganadería, la colocarán en la vanguardia de la "Nueva Revolución Verde", con un enfoque sustentable y amigable con los agroecosistemas.

\section{LITERATURA CITADA}

Bradfield SJ, Kumar P, White JC, Ebbs SD (2017) Zinc, copper, or cerium accumulation from metal oxide nanoparticles or ions in sweet potato: Yield effects and projected dietary intake from consumption. Plant Physiology and Biochemistry 110: 128-137.

Duhan JS, Kumar R, Kumar N, Kaur P, Nehra K (2017) Nanotechnology: The new perspective in precision agriculture. Biotechnology Reports 15: 11-23.

Gui X, Rui M, Song Y, Ma Y, Rui Y, Zhang P, Liu L (2017) Phytotoxicity of $\mathrm{CeO}_{2}$ nanoparticles on radish plant (Raphanus sativus). Environmental Science and Pollution Research 1-7: DOI 10.1007/s11356017-8880-1.

Ganesh, EN (2017) Application of Nanotechnology in Agriculture Sector-A Review. International Journal of Exclusive Global Research 1:12. 1-10.

Hill EK, Li J (2017) Current and future prospects for nanotechnology in animal production. Journal of Animal Science and Biotechnology 8: 1-13.

Lira-Saldivar RH, Ponce-Zambrano R, Ruiz-Torres NA, Méndez-Argüello B, Mendoza-Mendoza E, GarcíaCerda LA, Vera-Reyes I (2016) Synthesis of zinc oxide nanoparticles and its influence on seed germination and growth of Solanum lycopersicum seedlings. Wulfenia Journal 23: 220-235.

Swain PS, Rao SB, Rajendran D, Dominic G, Selvaraju S (2016) Nano zinc, an alternative to conventional zinc as animal feed supplement: A review. Animal Nutrition 2: 134-141.

Vankova R, Landa P, Podlipna R, Dobrev PI, Prerostova S, Langhansova L, Vanek T (2017) ZnO nanoparticle effects on hormonal pools in Arabidopsis thaliana. Science of the Total Environment 593: 535-542. 